\title{
Spontaneous Rupture of Urinary Bladder (SRUB) in a Young Male, Presenting as Anuria - A Case Report and Review of Literature
}

\author{
Sekar Hariharasudhan, Sriram Krishnamoorthy and Sunil Shroff*
}

Sri Ramachandra Medical College and Research Institute, Porur, Chennai, India

\begin{abstract}
Spontaneous rupture of urinary bladder is a rare condition. Most of the bladder ruptures occur in association with blunt or penetrating injuries to the lower abdomen. Most often, a vague lower abdominal pain is the mode of presentation. Rarely patients present with oliguria, anuria, uremia or urosepsis.

A forty year old male, under the influence of alcohol, presented with lower abdominal pain and anuria for two days with abnormal renal function. He denied history of blunt trauma. He was diagnosed to have an intra-peritoneal rupture of urinary bladder. He underwent emergency laparotomy with repair of bladder rupture.

This case report illustrates the need for a high index of clinical suspicion. Prompt diagnosis and appropriate management will help in preventing a poor clinical outcome in patients with spontaneous bladder perforation. If left untreated or if there is a considerable delay in diagnosis and intervention, it usually is associated with a high morbidity.
\end{abstract}

Keywords: Alcohol intoxication, anuria, bladder rupture.

\section{INTRODUCTION}

Most of the bladder injuries occur either due to blunt or penetrating injuries to the lower abdomen. Bladder injuries can also be iatrogenic. Spontaneous rupture of bladder is a relatively rare but potentially lethal condition which warrants an early diagnosis and appropriate management. A high index of suspicion is mandatory as this condition is often overlooked and conservatively managed as anuria or acute renal failure. We present a case of spontaneous rupture of urinary bladder in a young male which occurred after alcohol intoxication and presented to us with lower abdominal pain and anuria.

\section{CASE PRESENTATION}

A 40 year old male had diffuse lower abdominal pain and anuria for 2 days with initial passage of few drops of blood in urine. The following day he developed progressive abdominal distension and reported to a peripheral hospital where he was treated for the above complaints and subsequently referred to our emergency department.

On initial evaluation he was toxic with anuria of forty eight hours. Distinct bladder fullness could not be made out, as there was uniform lower abdominal distension. Ultrasound abdomen showed normal kidneys with ascites and an empty bladder. Both kidneys were found to be normal. He was immediately catheterized, but only $5 \mathrm{ml}$ of blood stained urine drained. Gentle bladder irrigation revealed a suspicion of bladder rupture. Computed

*Address correspondence to this author at the Department of Urology and Renal Transplantation, Sri Ramachandra Medical College and Research Institute, Porur, Chennai 600116, India; Tel: 00919840025680;

E-mail: srmcurology@gmail.com tomography (CT) scan of the abdomen and pelvis showed normal kidneys, but showed a focal discontinuity seen in the right superolateral aspect of the dome of the urinary bladder (Fig. 1). The tip of the Foley's catheter was seen lying outside the bladder (Fig. 2). There was a moderate fluid in the abdomen and pelvic cavity, raising the suspicion of urinary ascites. However his serum creatinine and total white blood cell count was normal. There was a fluctuant course of symptoms and urine output, with a difficulty in passing urine and on and off haematuria. However, he denied a history of blunt lower abdominal trauma. A diagnosis of spontaneous rupture of bladder was suspected. Retrograde cystogram confirmed the presence of intra peritoneal bladder rupture (Fig. 3). He was taken up for an emergency laparotomy. Preliminary cystoscopy demonstrated a large irregular tear of $6 \times 7 \mathrm{~cm}$ above and lateral to the right ureteric orifice and near

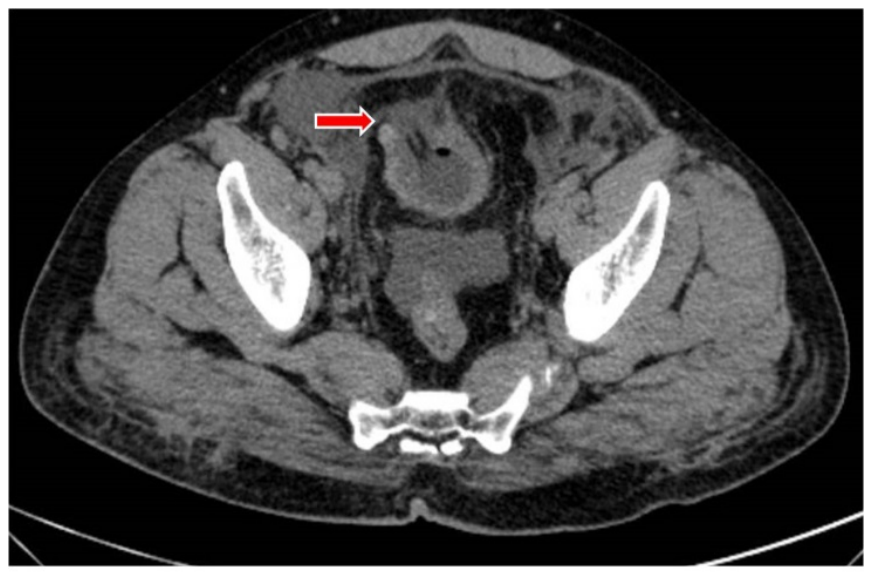

Fig. (1). CT pelvis showing the facial discontinuity in the dome of urinary bladder. 

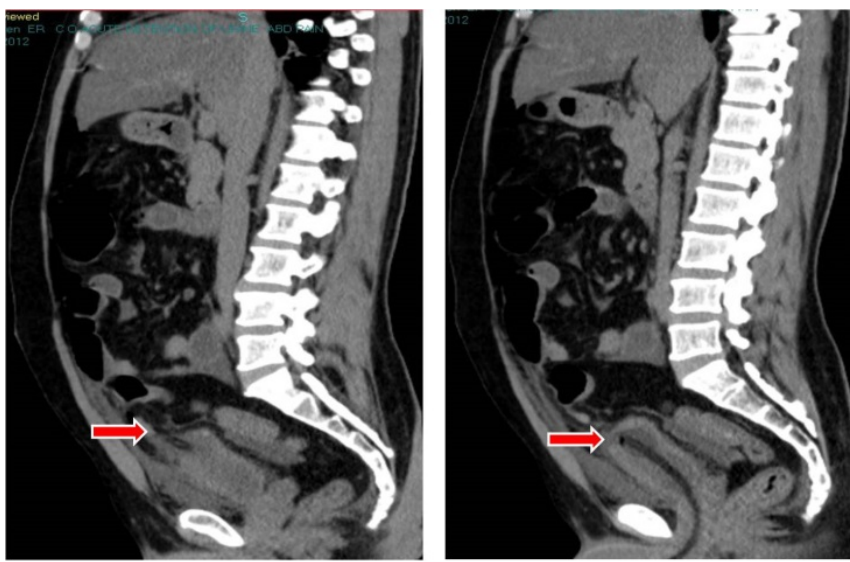

Fig. (2). CT pelvis showing the clip pf Foley catheter to be outside the bladder.
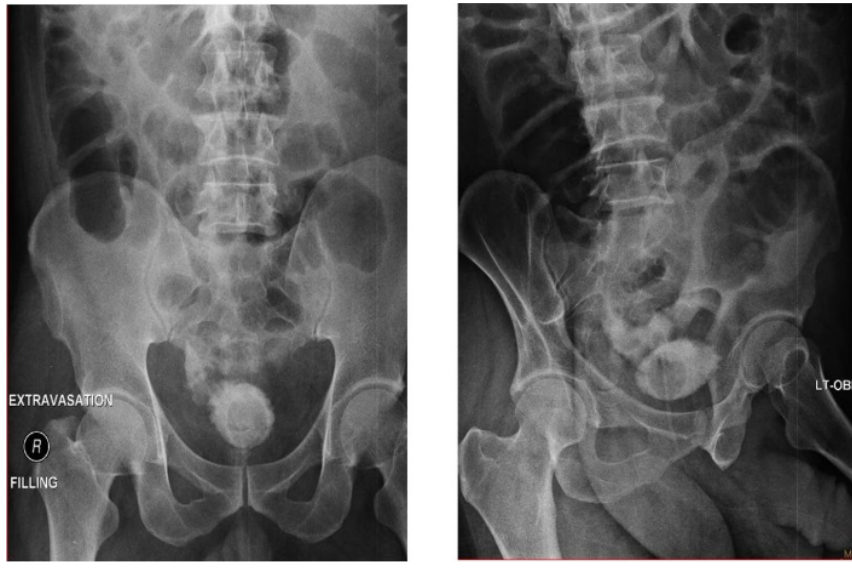

Fig. (3). Cystogram confirming the intra peritoneal rupture of the bladder.

the dome of the bladder (Fig. 4). Intra operatively about 4 liters of urine was drained from the peritoneal cavity. There was an irregular laceration in the postero superior aspect of the dome of the urinary bladder measuring $6 \times 7 \mathrm{~cm}$. The edges of the perforation were irregular, congested and edematous and friable. A biopsy of the margin of the

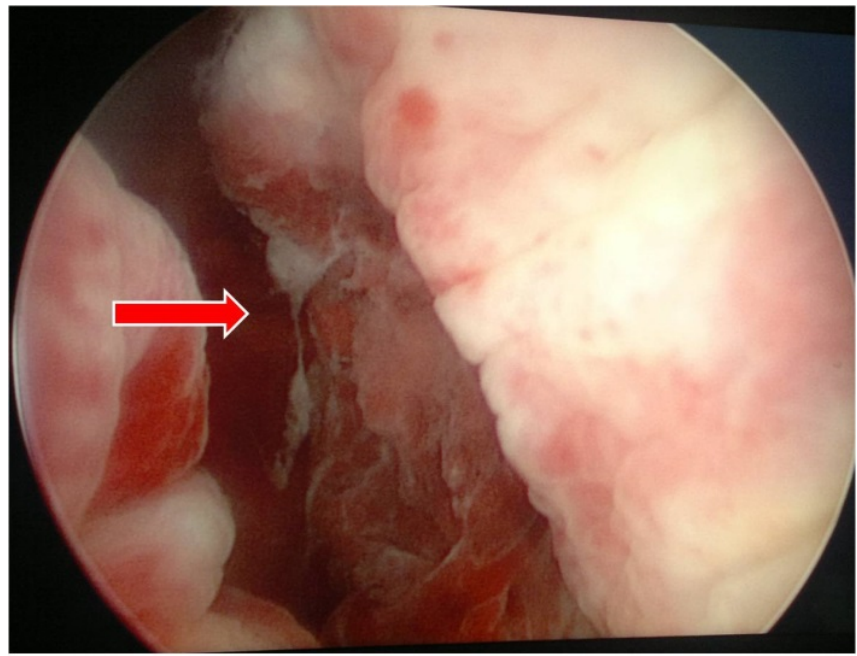

Fig. (4). Cystoscopy demonstrating the large irregular tear in the bladder. laceration was taken and three layered closure done with a separate suprapubic cystostomy and Foley's urethral catheter drainage. The histopathology report showed only inflammatory changes. The individual made an uneventful postoperative recovery (Fig. 5).

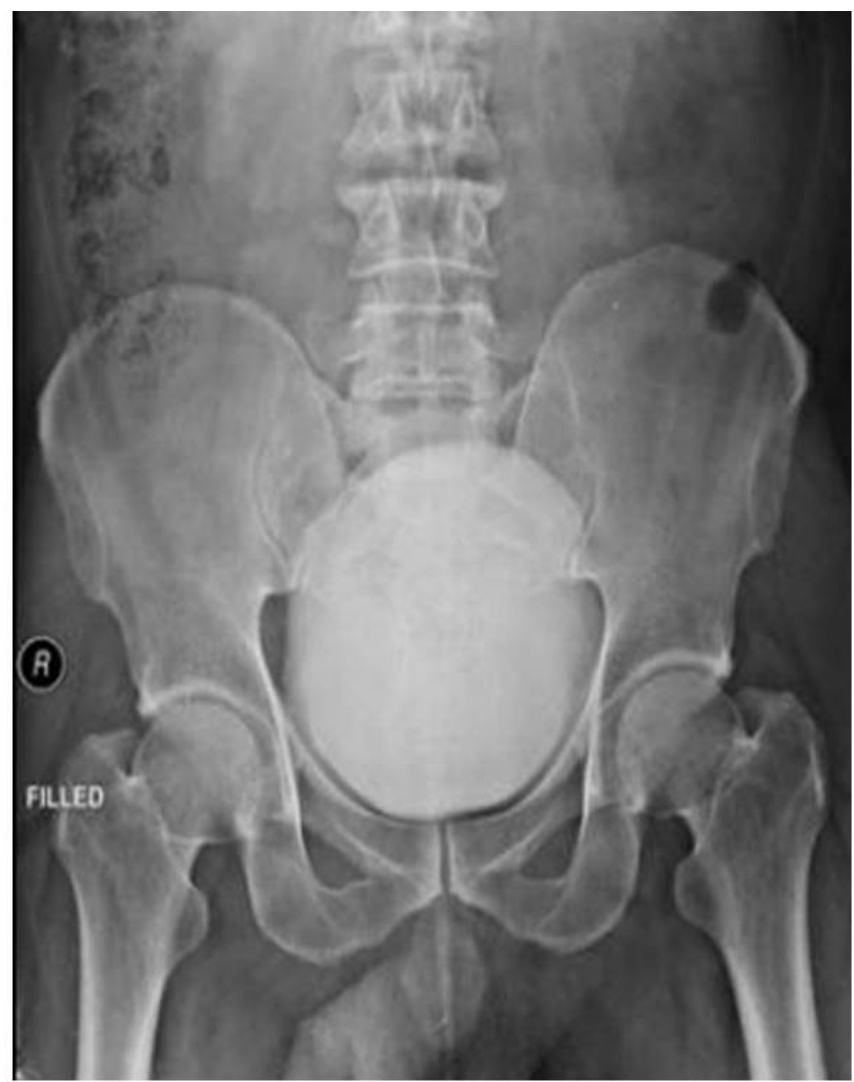

Fig. (5). Post operative cystogram.

\section{DISCUSSION}

SRUB is a rare condition. Bastable JR et al. have reported an overall incidence of 1:126000 [1]. Most of them occur in males (79\%) [2]. Alcohol abuse resulting in a bladder rupture has been reported in a very few cases [3]. SRUB has been reported not only with alcohol consumption but also with other substance abuse like cocaine and amphetamines [4]. It is also known to occur in malignancies of the urinary bladder, neurogenic bladder or post irradiation bladders. Jenkinson et al. proposed a classification of spontaneous bladder rupture, wherein he had classified them as due to Idiopathic, drug induced, obstructive and due to bladder wall lesions [5].

Budd et al. in 1988 reported his case of spontaneous bladder rupture in a female with transitional cell carcinoma of the bladder [6]. Glashan et al. attributes the male predominance to the anatomical features of the male urethra that makes the male bladders more liable to distension and a consequent rupture [7].

Jamil Ahmed et al. in his review of literature of spontaneous bladder rupture identified that only 2 of the 15 cases reported had an accurate pre operative diagnosis, while the rest were initially managed conservatively with catheterization and antibiotics [8]. 
Lutwak reported a rare presentation of a 32 year old diabetic male who presented with bladder rupture and urosepsis following TURBT [9]. Various others have reinforced this particular etiology of diabetes causing decreased bladder sensitivity due to autonomic neuropathy leading to chronic urinary retention and recurrent infection which might predispose to spontaneous bladder rupture.

The basic pathogenesis of SRUB involves bladder over distension with thinning of the dome of the bladder from diuresis. Under alcohol influence, patient ignores the desire to void, resulting in bladder over distension. Subsequently, even a further trivial rise in the intra abdominal pressure can cause rupture of the thinned out and over distended bladder. The patient develops severe suprapubic pain with progressive abdominal distension due to urinary ascites with reverse auto dialysis wherein urea and creatinine molecules are absorbed into the blood mimicking pseudo renal failure [10]. While most ruptures are intraperitoneal with resultant urinary ascites, extra peritoneal ruptures have also been described. The most important key to an effective and efficient management of such cases is a thorough knowledge of such a condition and a high index of clinical suspicion.

CT cystogram is mostly diagnostic, but it is expensive and may not be readily available. Conventional cystogram is also equally effective in diagnosis. Retrograde cystography is the standard diagnostic procedure in the evaluation of bladder trauma. This is the most accurate radiological study to identify bladder rupture. When bladder filling and post void images are obtained, cystography has an accuracy rate of $85-100 \%$ [11]. Two or three layered bladder closure with a separate suprapubic cystostomy is recommended. A bladder biopsy, to exclude other pathology, is also recommended [12]. If detected late, this condition is associated with a high mortality rate of $50 \%$ [13].

\section{CONCLUSION}

A very high index of clinical suspicion is mandatory in making a diagnosis of spontaneous bladder ruptures. This diagnosis should be considered in those patients, who present with an acute abdomen and anuria with elevated serum creatinine levels. These cases are initially managed by general surgeons and emergency department medical staffs.
Hence it is very important to make them be aware of this condition as one of the differential diagnosis in patients with acute abdomen. Appropriate diagnosis and prompt management would prevent a poor clinical outcome in such cases.

\section{CONFLICT OF INTEREST}

The authors confirm that this article content has no conflict of interest.

\section{ACKNOWLEDGEMENTS}

Declared none.

\section{REFERENCES}

[1] Bastable JR, De Jode LR, Warren RP. Spontaneous rupture of the bladder. Br J Urol 1959; 31: 78-86.

[2] Glashan RW. Perforation as a complication of carcinoma of the bladder. Br J Urol 1967; 39: 178-80.

[3] Dooldeniya MD, Khafagy R, Mashaly H, Browning AJ, Sundaram SK, Biyani CS. Lower abdominal pain in women after binge drinking. BMJ 2007; 335: 992-3.

[4] Bennett AH, Delrio A. Idiopathic rupture of the bladder: association with methamphetamine and alcohol. J Urol 1980; 124 429-30.

[5] Jenkinsonlr. Spontaneous intraperitoneal rupture of the urinary bladder. Postgrad Med J 1981; 57: 269-70.

[6] Budd JS. Spontaneous intraperitoneal rupture of the bladder in association with transitional cell carcinoma. Postgrad Med J 1988; 64: $165-6$.

[7] Glashan RW. Perforation as a complication of carcinoma of the bladder. Br J Urol 1967; 39: 178-80.

[8] Ahmed J, Mallick IH, Ahmad SM. Rupture of urinary bladder: a case report and review of literature. Cases J 2009; 2: 7004.

[9] Lutwak N, Dill C. Urosepsis complicated by a spontaneous bladder perforation. BMJ Case Rep 2011.

[10] Chow KM, Lam CM, Szeto CC. Pseudo-renal failure following total abdominal hysterectomy. J Nephrol 2005; 18: 442-6.

[11] N Djakovic, E Plas, L Martínez-Piñeiro, et al. Members of the European Association of Urology (EAU) Guidelines Office. Guidelines on urological trauma. In: EAU Guidelines, edition presented at the 24th EAU Annual Congress, Stockholm 2009. ISBN 978-90-79754-83-0

[12] Lt Col AA Pradhan: Spontaneous rupture of bladder: a rare clinical entity. MJAFI 2007; 63: 92-93.

[13] Lynn SJ, Mark SD, Searle M. Idiopathic spontaneous bladder rupture in an intoxicated patient. Clin Nephrol 2003; 60: 430-2. 